\title{
Allan Botkin Responds
}

To the Editor:

I thank Bruce Horacek for having taken an interest in my article. I welcome the opportunity to provide further clarification.

First, the findings I presented were derived from clinical observations, not from research. My goal in publishing the article was simply to share the induction technique with others. I have some confidence that other therapists trained in eye-movement desensitization and reprocessing (EMDR) who follow the procedure as described will 
achieve similar outcomes. So far, interested colleagues have been able to induce after-death communications (ADCs) reliably after some instruction. My hope is that other professionals will test my clinical observations in a more rigorous and scientific manner.

Second, I am aware that my conclusions are not consistent with current thinking in the field. However, my ideas are new, not old. Although I do not believe that a sense of loss is generally time limited, I used the terms grief and loss in an interchangeable manner because, when it comes to ADCs induced with EMDR, there is no difference. Both acute grief and a lifelong sense of loss respond equally well to the procedure. In fact, a high percentage (more than 40 percent) of my cases (more than 500) are clearly not acute grief reactions, and in these cases, there are also no associated symptoms of posttraumatic stress disorder. These patients were treated for a sense of loss that continued for many years (sometimes decades) after they had successfully worked through their acute phase of grief. In short, whether acute grief or a protracted sense of loss is involved, grief/loss is resolved by an ADC induced with EMDR.

An important distinction will perhaps further clarify this issue. I now regret not including this discussion in my article. I believe that there are two levels of a sense of loss. The more painful and deeper level is a feeling of disconnection, a realization or feeling that our loved one is gone. ADCs induced with EMDR provide survivors with an experiential reconnection that resolves this deep pain. It should be obvious, however, that even in these cases, nothing can bring our loved one back to life as we generally know it, and certain life experiences, such as waking up in the morning together or holidays, can no longer be shared in the usual way. The loss of these shared activities does not fully resolve with an ADC. However, having resolved the deeper sense of loss with an induced ADC, the loss of shared activities, even at the most difficult times, becomes more tolerable, and survivors are able to experience positive feelings and memories of the deceased, as well as more fully enjoy their relationships with surviving loved ones.

I am grateful that Horacek's well-intended and scholarly criticisms provided me an opportunity to further clarify a very important aspect of my report. Although all prior feedback has been positive, I expect that some future responses may be less well-intended, and perhaps some even hostile. Skepticism will take a variety of forms. Nevertheless, I feel compelled to take the risk. It seems to me that EMDR-induced $\mathrm{ADCs}$ have the potential to alleviate a great deal of suffering. It is my 
hope that others, both EMDR trained therapists and clients/patients, will put their beliefs on hold long enough to give it a try.

Allan L. Botkin, Psy.D.

1144 Harms

Libertyville, IL 60048

e-mail: DrAl53@aol.com 